\title{
Anesthetic management of a known case of Raynaud's phenomenon posted for breast conservative surgery- a case report
}

\author{
Kalpajit Dutta ${ }^{1 *}$, Shalini Saini' ${ }^{1}$, Sushma Bhatnagar ${ }^{1}$ and S.V.S. Deo ${ }^{2}$ \\ ${ }^{1}$ Department of Anesthesiology, Pain \& Palliative Care, BRA-IRCH, AIIMS, New Delhi, India \\ ${ }^{2}$ Department of Surgical Oncology, BRAIRCH, New Delhi, India
}

\begin{abstract}
Raynaud's phenomenon is a disorder of the microvasculature that generally affects the fingers and toes but can present on other extremities such as the nose, ears and nipples etc. which occurs as a result of vasoconstriction of the digital arteries, arterioles, and cutaneous arteriovenous shunts. The hallmark of Raynaud's phenomenon is ischemia of the digits in response to cold, which produces a characteristic "triphasic" color pattern. The balance of vasodilatation and vasoconstriction is always required to maintain adequate tissue perfusion which is tightly regulated by local control mechanisms as well as neuro-hormonal mediators. If the vasospasm is severe and long-lasting, the attack may lead to critical ischemia and necrosis of the digits. Raynaud's phenomenon may occur after administration of B-adrenoceptor blockers or chemotherapeutic agents like vinblastine and bleomycin. Localized vasospasm may occur after the use of vasoconstrictor drugs, intra-arterial or extravasation of anaesthetic agents like thiopental, diazepam, phenytoin etc. Here we present the perioperative concerns and precautions that need to be taken in the management of a known case of Raynaud's phenomenon.
\end{abstract}

\section{Introduction}

Raynaud's phenomenon, named after the French physician Maurice Raynaud, is a disorder of the microvasculature that generally affects the fingers and toes but can present on other extremities such as the nose, ears and nipples [1-3]. Raynaud's phenomenon occurs as a result of vasoconstriction of the digital arteries, arterioles, and cutaneous arteriovenous shunts [4]. It was further divided into primary Raynaud's disease and secondary Raynaud's phenomenon. Secondary Raynaud's phenomenon is often related to connective tissue disorders, but also to physical and chemical strain. Though the pathophysiology of Raynaud's phenomenon is still not well understood, systemic and local vascular effects are most likely to be associated with primary Raynaud's disease Additional abnormalities in vascular structure and function also may play a major role in secondary Raynaud's phenomenon. Regardless of the subtype, the hallmark of Raynaud's phenomenon is ischemia of the digits in response to cold, which produces a characteristic "triphasic" color pattern (white to blue to red) as well as swelling and numbness $[2,5]$. Initially, the distal parts fingers become pale or white due to constricted blood-flow; then it become blue, which is a sign of tissue hypoxia; and lastly red, when the tissue is reperfused $[3,6]$. Raynaud's phenomenon may occur after administration of B-adrenoceptor blockers or chemotherapeutic agents like vinblastine and bleomycin $[7,8]$. Although localised vasospasm may occur after intra-arterial or extravasation of anaesthetic agents like thiopental, diazepam, phenytoin etc. Raynaud's phenomenon has not been described as a complication of anaesthesia. To the best of our knowledge there is no case report of anesthetic management of patient with raynaud's phenomenon in literature. We present the perioperative management of a known case of Raynaud's phenomenon in an otherwise healthy patient posted for breast conservative surgery.

\section{Case history}

A 42 year, female patient posted for left breast conservative surgery for cancer breast. She had history of hypersensitivity to cold since last 20 years. She had history of blanching of hands followed by bluish discolouration and redness on exposure to cold water associated with pain in the limbs. She also noticed 4-5 similar episodes of skin reaction after she went out in rain in last 5 years. These symptoms are sometimes associated with vomiting. She had her last episode in August 2014 during river rafting. She has always been using warm water to take bath, warm food to eat and avoids air conditioned room for last 20 years. There was no family history of similar complaints. She was not on any medications for the complaints. She had no history of drug allergy. There is no surgical history. Preoperatively routine investigations were within normal limits. Preoperative preparation included increasing operation theatre temperature, fluid warmer and warming blankets were kept ready (Figure 1). 10\% lignocaine topical spray was applied on the dorsum of hand of interest for cannulation (Figure 2) (as EMLA cream was not available), followed by warming the localized area with the help of warm water filled glove. After preparation of hand, 20G IV cannula was secured on dorsum of right hand. Warm intravenous fluid was used intraoperatively. Fentanyl 80 microgram was used for analgesia. Anesthesia was induced with injection propofol $100 \mathrm{mg}$, neuromuscular relaxation achieved with injection vecuronium $8 \mathrm{mg}$.

Correspondence to: Kalpajit Dutta, Department of Anesthesiology, Pain and Palliative Care, BRA-IRCH, AIIMS, New Delhi, India, Tel: 9958021172; E-mail: kalpajit.dutta@gmail.com

Key words: ischemia, necrosis, raynaud's phenomenon, vasospasm

Received: July 22, 2015; Accepted: August 24, 2015; Published: August 28, 2015 


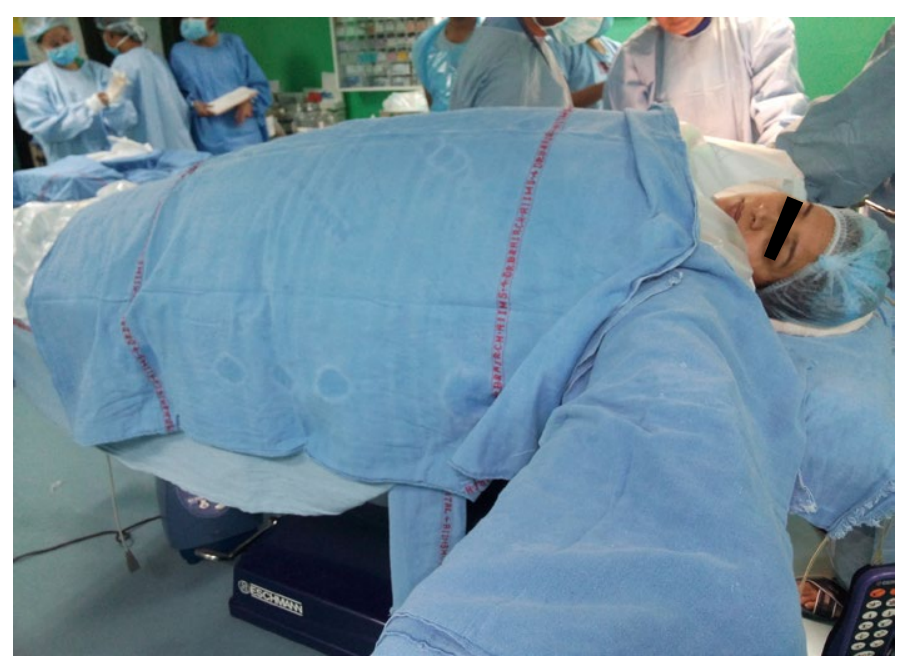

Figure 1. Maintaining proper warmth to the patient.

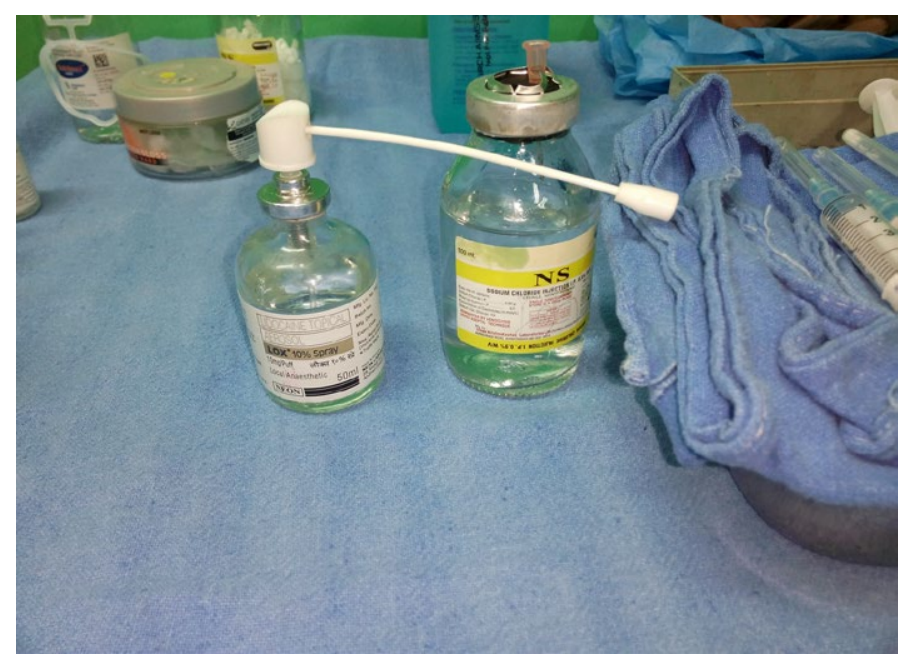

Figure 2. 10\% Lignocaine spray before IV cannulation and warm normal saline for drug dilution.

Airway was secured with I-gel size 4 . Anesthesia was maintained with oxygen- air mixture in $4 \%$ sevoflurane with low flows. Intraoperatively, surgical aseptic preparation was done with warm solution of scrub betadine. Dilution of drugs was done with warm normal saline (Figure 2). No episode of color change in extremities noted (Figure 3). Hemodynamic parameters remained stable and within normal limits (Figures 4 and 5) throughout the surgical procedure (Figure 6). The total anaesthesia time was 90 minutes. The patient was observed in the recovery room for 2 hour. Thereafter, patient was shifted to the surgical ward. Routine investigations on the next day revealed no abnormality. Patient was discharged on the next day.

\section{Discussion}

Raynaud's phenomenon is an exaggerated vasospastic response to cold or to emotional stress that can be either primary (idiopathic) or secondary to a number of underlying conditions such as connective tissue disorders, vascular obstructions or drugs. Raynaud's phenomenon represents as a failure of acral vasomotor auto-regulation. The balance of vasodilatation and vasoconstriction is always required to maintain adequate tissue perfusion which is tightly regulated by local control mechanisms as well as neuro-hormonal mediators. If the vasospasm is severe and long-lasting, the attack may lead to critical ischemia and necrosis of the digits.

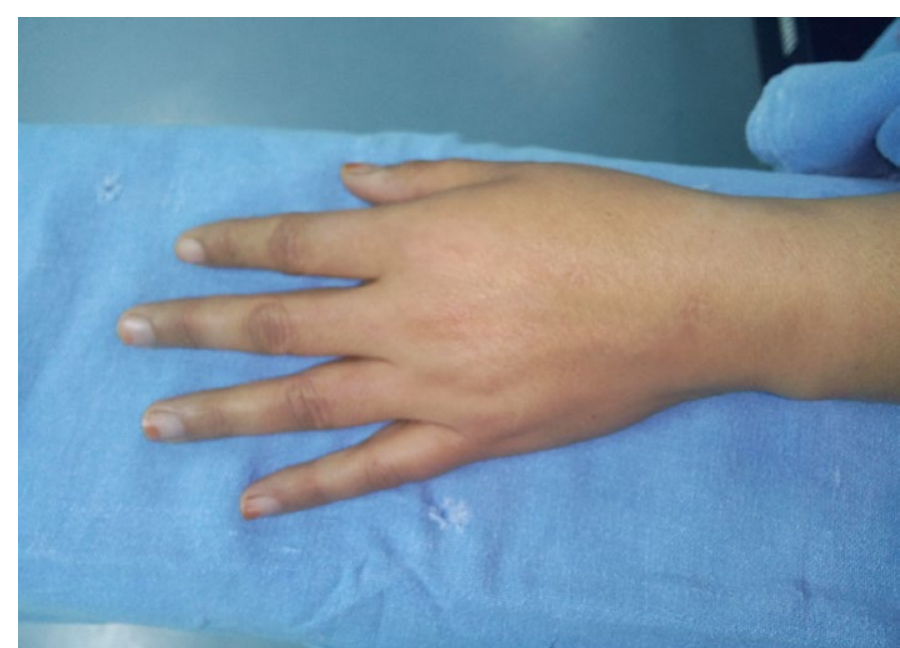

Figure 3. Normal skin colour of the patient at room temperature.

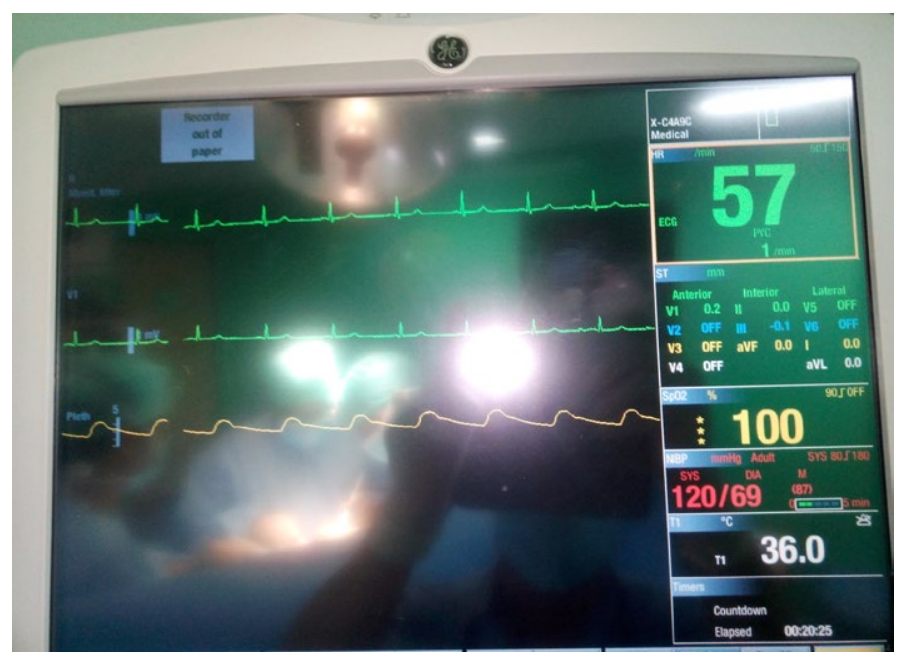

Figure 4. Intra-operative stable hemodynamics.

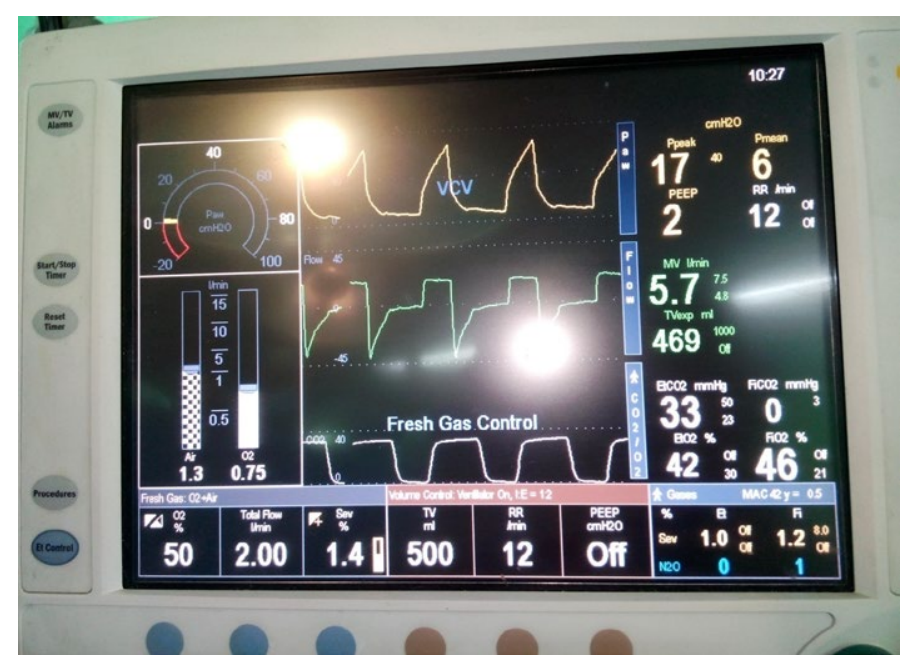

Figure 5. Intra-operative stable ventilator parameters. 


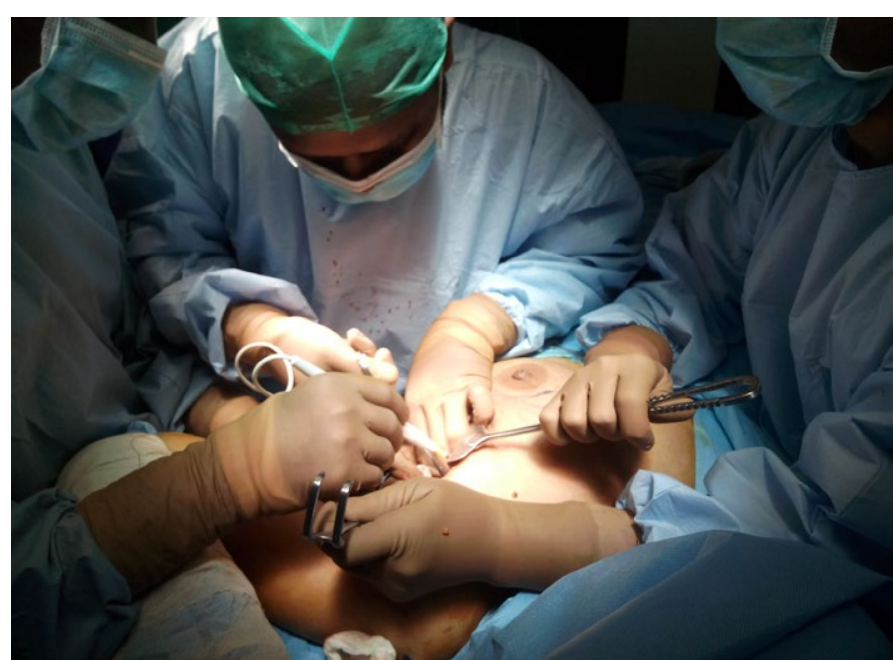

Figure 6. Intraoperative steps of breast conservative surgery.

Table 1. Precautions that need to be taken in a case of known Raynaud's phenomenon.

Quit smoking

keep the whole body warm, especially hands and feet

Patient should wear gloves and warm footwear in cold weather

Regular exercise helps to improve circulation and reduce stress levels

Relaxation techniques such as yoga, deep breathing exercises to minimise stress levels

To avoid stimulants such as such as coffee, tea and cola

Avoid sympathomimetic medications and emotional stress

To wear gloves or mittens when taking food out of the refrigerator

Air conditioner should be set to a warmer temperature.

Moving to a warmer climate may help people with severe Raynaud's phenomenon.

\section{Complications of raynaud's phenomenon are}

1. There could be permanent reduction of blood circulation to the fingers or toes which can cause deformities of the fingers or toes.

2. If an artery to an affected area becomes blocked completely, sores (skin ulcers) or dead tissue (gangrene) may develop.

3. The symptoms of gangrene normally begin with a red line on the skin which marks the edges of the affected tissue. The affected tissue becomes cold and numb. It can be painful when the tissue dies.

4. There is colour change in the affected area from red to brown to black. The dead tissue will gradually shrink in size, separate from the healthy tissue and finally fall off.

5. In extreme untreated cases affected part of the body may need to be removed (amputation).

Beta-adrenoceptor blocking agents are known to cause coldness of the extremities, and vasoconstrictors such as ergotamine are contraindicated in Raynaud's syndrome [9]. Mohokum M et al. in their meta analysis found that the prevalence of raynaud's phenomenon is more in patients receiving beta blockers [10]. Csiki $\mathrm{Z}$ et al. found in their study that the concurrent administration of beta-blockers with calcium channel blockers reduces symptoms in patients suffering from Raynaud's syndrome [11]. Chemotherapeutic agents like bleomycin, cisplatin, vinblastine are reported to induce Raynaud's phenomenon, but yet the underlying mechanism is unclear $[7,8,12]$. Although accidental intra-arterial injection or extravasation of anaesthetic agents like thiopental,diazepam, phenytoin etc may cause localised vasospasm, Raynaud's phenomenon has not been described as a complication of anaesthetic drugs. Emanual Maverakis et al. agreed that white/pallor and blue/cyanosis were the two most important colors to make a diagnosis of raynaud's phenomenon and that patients must report cold temperatures as one of the triggers for their Raynaud's phenomenon attacks. They were also in agreement that a normal ESR and a normal ANA were not required for a diagnosis of primary Raynaud's phenomenon [13]. In our case the patient has typical presentation of colour change of Raynaud's phenomenon and ANA tests were not done. As cold is a strong stimulus for Raynaud's attack we have maintained a warm operation theatre temperature as well as all IV fluids and wash saline were prewarmed. Exposed areas were properly covered with cotton pads. N.M. Bedforth et al. (1995) noticed in their case that the area treated with EMLA cream for putting IV line prior to induction was completely unaffected by the intraoperative vasospastic episode. As an alternate to EMLA cream, we sprayed the area with $10 \%$ lignocaine that provided equal effect. Warming the area of interest achieved by placing warm water filled glove in hand of the patient. All forms of drugs which might cause vasoconstriction such as phenylephrine, mephentermine, epinephrine, nor-epinephrine, diazepam, thiopental, phenytoin etc were avoided. Precautions that need to be taken in a case of known Raynaud's phenomenon are also mentioned in table 1 .

Following a substantial review of literatures, we found this to be the only reported case of anesthetic management of a known case of Raynaud's phenomenon.

\section{Conclusion}

Though the pathophysiology of Raynaud's phenomenon is still not well understood, systemic and local vascular effects are mostly associated with primary Raynaud's disease. Intraoperative use of multiple drugs, IV fluids, saline wash and other stress factors can lead to vasoconstrictions resulting in aggravation of preexisting disease. Therefore vigilance of the anesthesiologist is very much required in intraoperative period.

\section{References}

1. Anderson ME, Allen PD, Moore T, Hillier V, Taylor CJ, et al. (2005) Computerized nailfold video capillaroscopy--a new tool for assessment of Raynaud's phenomenon. $J$ Rheumatol 32: 841-848. [Crossref]

2. Block JA, Sequeira W (2001) Raynaud's phenomenon. Lancet 357: 2042-2048 [Crossref]

3. Goundry B, Bell L, Langtree M, Moorthy A (2012) Diagnosis and management of Raynaud's phenomenon. BMJ 344: e289. [Crossref]

4. Wigley FM (2002) Clinical practice. Raynaud's Phenomenon. N Engl J Med 347 1001-1008. [Crossref]

5. Olsen N (1988) Diagnostic tests in Raynaud's phenomena in workers exposed to vibration: a comparative study. Br J Ind Med 45: 426-430. [Crossref]

6. Cooke JP, Marshall JM (2005) Mechanisms of Raynaud's disease. Vasc Med 10: 293 307. [Crossref]

7. Doll DC, Yarbro JW (1992) Vascular toxicity associated with antineoplastic agents Semin Oncol 19: 580-596. [Crossref]

8. Hansen SW, Olsen N, Rossing N, Rørth M (1990) Vascular toxicity and the mechanism underlying Raynaud's phenomenon in patients treated with cisplatin, vinblastine and bleomycin. Ann Oncol 1: 289-292. [Crossref]

9. British Medical Association and Royal Pharmaceutical Society of Great Britain (1994) British National Formul iry 27: 184.

10. Mohokum M, Hartmann P, Schlattmann P (2012) The association of Raynaud syndrome with $\hat{\mathrm{I}}^{2}$-blockers: a meta-analysis. Angiology 63: 535-540. [Crossref]

11. Csiki Z, Garai I, Shemirani AH, Papp G, Zsori KS, et al. (2011) The effect of metoprolo 
Dutta K (2015) Anesthetic management of a known case of Raynaud's phenomenon posted for breast conservative surgery- a case report

alone and combined metoprolol-felodipin on the digital microcirculation of patients with primary Raynaud's syndrome. Microvasc Res 82: 84-87. [Crossref]

12. Heier MS, Nilsen T, Graver V, Aass N, Fossa-SD (1991) Raynaud's phenomenon after combination chemotherapy of testicular cancer, measured by laser Doppler flowmetry. A pilot study. British Journal of Cancer 63: 550-552. [Crossref]

13. Maverakis E, Patel F, Kronenberg DG, Chung L, Fiorentino D, et al. (2014) International consensus criteria for the diagnosis of Raynaud's phenomenon. J Autoimmun 48-49: 60-65. [Crossref]

Copyright: (C2015 Dutta K. This is an open-access article distributed under the terms of the Creative Commons Attribution License, which permits unrestricted use, distribution, and reproduction in any medium, provided the original author and source are credited. 